\title{
Combined Bidding at Power and Ancillary Service Markets
}

\author{
A. Virag ${ }^{\# 1}$, A. Jokić ${ }^{\# 2}$, R.M. Hermans ${ }^{\# 3}$, P.P.J. van den Bosch ${ }^{\# 4}$ \\ \# Department of Electrical Engineering, Eindhoven University of Technology \\ Den Dolech 2, 5612 AZ Eindhoven, The Netherlands \\ ${ }^{1}$ a.virag@tue.nl, ${ }^{2}$ a.jokic@tue.nl, ${ }^{3}$ r.m.hermans@tue.nl, ${ }^{4}$ p.p.j.v.d.bosch@tue.nl
}

\begin{abstract}
Power and ancillary service markets are strongly coupled. However, at the moment auctions are organized in such a manner that the coupling is not taken into account. Every market participant submits bids at power and/or AS markets without possibility to adjust with respect to the outcome of the other market. Inexact approximations of the actual market price induce deviations from the optimal social welfare value.

In this paper, we firstly describe and analyze the consequences of power and ancillary service market coupling. Secondly, we present two different market design strategies, both of which are based on the idea of iterative auction and have the goal to optimally account for power and AS coupling, enabling the overall system to maximize its social welfare.

An illustrative example is used to present potential benefits and downsides that might arise as a result of introducing proposed market arrangements.
\end{abstract}

\section{NOMENCLATURE}

$J_{i} \quad$ is an objective function in a node $i, i=1, \ldots, n$

$\mu_{i} \quad$ denotes a probability of activating allocated reserves, $0 \leq \mu_{i} \leq 1$

$p:=\operatorname{col}\left(p_{1}, \ldots, p_{n}\right)$ is a vector ${ }^{1}$ of power injections; depending on the sign it can represent produced $(+)$ or consumed (-) power

$a:=\operatorname{col}\left(a_{1}, \ldots, a_{n}\right)$ is a vector of allocated reserve power; always positive

$A_{\text {req }}$ is a required amount of reserves in a control zone

$\bar{p}:=\operatorname{col}\left(\overline{p_{1}}, \ldots, \overline{p_{n}}\right)$ is a suitably defined vector of maximal power injections at the nodes

$\underline{p}:=\operatorname{col}\left(\underline{p_{1}}, \ldots, \underline{p_{n}}\right)$ is a vector of minimal power injections at the nodes

$p^{*}:=\operatorname{col}\left(p_{1}^{*}, \ldots, p_{n}^{*}\right)$ is a vector of optimal nodal power injections

$a^{*}:=\operatorname{col}\left(a_{1}^{*}, \ldots, a_{n}^{*}\right)$ is a vector of optimal nodal reserve power allocations

$\lambda_{\mathrm{P} i}\left(p_{i}\right)$ is a bid of a $\mathrm{BRP}_{i}{ }^{2}$ to power market

$\lambda_{\mathrm{A} i}\left(a_{i}\right)$ is a bid of a $\mathrm{BRP}_{i}$ to $\mathrm{AS}$ market

$\lambda_{\mathrm{P}}^{(k)}$ is a global power price in iteration $k$

$\lambda_{\mathrm{A}}^{(k)}$ is a global AS price in iteration $k$

\section{INTRODUCTION}

A stable, reliable and economically efficient electric energy supply plays a crucial role in every modern society. The

\footnotetext{
${ }^{1}$ We use $\operatorname{col}\left(x_{1}, \ldots, x_{n}\right)$ to denote the column vector $\left(\begin{array}{lll}x_{1} & \ldots & x_{n}\end{array}\right)^{\top}$.

${ }^{2}$ BRP stands for "Balance responsible party" and is a market participant acting on both, power and ancillary service, markets.
}

liberalization of electricity markets started a few decades ago with the goal of improving the economic efficiency of power systems (by this, we mean increasing the social welfare, i.e., the sum of benefits for producers and consumers). Compared to other commodities, power has several specific characteristics. To name a few: $i$ ) the inability of efficiently storing large energy quantities, which, as a consequence, leads to ii) the necessity of meeting the demand by production in real-time (power balancing); and iii) the lower demand priceelasticity of power as a commodity. For all these reasons, power markets differ from standard markets, making their design a challenging topic.

To facilitate real-time power balancing, other than power markets, another type of markets was created - ancillary services (AS) markets. On these markets, participants are paid for availability and/or ${ }^{3}$ producing more (or less) power than contracted to assist the system operation. Power and AS markets are strongly coupled and actions taken on one of the markets affect the other. The coupling comes from the fact that both commodities, power and AS, are provided by the same set of units that have certain production and consumption limits. Coupling also results from using the same limited transmission system in which power flows obey the laws of physics (Kirchoff's law) and are, in general, not controllable.

Conventional day-ahead power markets are based on collecting 24 hour power production and consumption profiles from all participants, i.e., balance responsible parties (BRP). We define a BRP as the only entity allowed to participate in power and AS markets. These power profiles are discrete in time, i.e., they are divided into a number of program time units (PTU). After all BRPs bid their offers or needs, one entity, a power exchange (PX), aggregates them in one common power supply and one common power demand curve and clears the market. Afterwards, BRPs can offer their spare capacity to a transmission system operator (TSO) on AS markets and thus potentially earn more money. ${ }^{4}$ Therefore, power and AS markets are, at the moment, organized in such a way that each BRP has to submit two independent bids, not knowing the

\footnotetext{
${ }^{3}$ In the majority of current arrangements, participants are paid only for producing (reducing) extra power; however, there are some countries, such as Norway, in which they are rewarded not only for producing more (or less) than contracted, but also for being available.

${ }^{4}$ Bids can afterwards be slightly changed on an intra-day power market, but this is outside the scope of this paper.
} 
result of one market when bidding for the other. This means that during the bidding phase, the coupling of power and AS markets is not taken into account neither on a BRP (local) nor on a global level.

It is indisputable that introducing markets in power systems has increased economic efficiency, see e.g. [1]. Still, it has been recognized that, due to the above explained independence of submitting bids, the markets designed in the current manner do not necessarily reach optimal economic efficiency[2]. In other words, current arrangements for power trading do not, in general, guarantee reaching the optimal social welfare value, even under conditions of perfect market competition when BRPs bid their marginal costs. This is due to necessary approximations that market participants have to make while submitting the bids to power and AS markets. So far, the research that aimed at solving the implications induced by market coupling, concentrated on bidding strategies. On the other hand, the research that aimed at increasing the social welfare investigated optimization techniques, but not in the view of iterative market auctions [3], [4], [5]. One of the first papers that proposed iterative decentralized markets is [6]. There, the authors describe a decentralized market pool auction for real and reactive power. However, in their work, the coupling of energy and AS markets is not considered.

In this paper, we present the results of a study on possibilities of increasing the economic efficiency of power markets by changing the way of bidding. We investigated the benefits of introducing iterative markets to overcome the BRP's problems of making approximations that prevent the overall system from reaching the optimal point. We proposed and compared two different market structures for solving the problem, which is formalized in Section III, both based on the idea of iterative markets and presented in detail in Section IV-C and Section IV-B. Our preliminary results show that iterative markets are well worth exploring in more detail as their introduction would eliminate the effects of errors in prediction of market outcomes for BRPs. It would also increase the overall social welfare.

\section{PROBLEM FORMULATION}

The problem under consideration is given in Problem III.1 and is referred as "optimal power and reserve dispatch" (OPRD) problem.

\section{Problem III.1 The Optimal Power and Reserve Dispatch (OPRD) Problem}

$$
\min _{p, a} J(p, a)=\min _{p, a} \sum_{i=1}^{n} J_{i}\left(p_{i}, a_{i}\right)
$$

subject to:

$$
\begin{aligned}
\sum_{i=1}^{n} p_{i} & =0 \\
\sum_{i=1}^{n} a_{i} & \geq A_{\text {req }} \\
\underline{p_{i}+a_{i}} & \leq p_{i} \leq \quad \overline{p_{i}}-a_{i}, \quad \forall i \in\{1, \ldots, n\} \\
a_{i} & \geq 0,
\end{aligned}
$$

where $J_{i}\left(p_{i}, a_{i}\right)=\left(1-\mu_{i}\right)\left(\alpha_{i} p_{i}^{2}+\beta_{i} p_{i}\right)+\mu_{i}\left[\alpha_{i}\left(p_{i}+a_{i}\right)^{2}+\right.$ $\left.\beta_{i}\left(p_{i}+a_{i}\right)\right]$.

The objective function $J_{i}$ is assumed to be a convex function. In case $\mathrm{BRP}_{i}$ in a node $i$ is a producer, $J_{i}$ represents the corresponding production cost. In case $\mathrm{BRP}_{i}$ in a node $i$ is a consumer, $J_{i}$ is the corresponding negated benefit function. Note here that $J$, as defined above, has an interpretation of a social welfare function.

For the sake of simplicity, we have chosen $\mu_{i}$ to be a parameter that indicates the probability of activating all allocated reserves. Such a choice of $\mu_{i}$ implies that, with respect to activating allocated reserves, there are only two possible cases considered: $i$ ) reserves are not activated at all, or ii) reserves are activated, and they are activated in their full allocated amount. Constraint (2a) denotes the power balance requirement, while ( $2 \mathrm{~b})$ means that there should always be a sufficient amount of reserves, $A_{\text {req }}$, in the system. The amount $A_{\text {req }}$ is chosen to be deterministic and known in advance, as it is in current market arrangements ${ }^{5}$. Equation (2c) states that every producer or consumer has production (and consumption) capacity limits, i.e., there are bounds on power injections in each node. Constraints (2d) come from another simplification of the presentation. Note that in $(2 \mathrm{~d})$ we have assumed the symmetry between the up-regulating AS and down-regulating AS. In a more general setting, the up-regulating limit and down-regulating limit can be different, and up-regulating and down-regulating actions can be treated as different commodities in AS markets. Results presented in this study can be easily generalized in that direction.

The OPRD problem can be seen as a variation of the optimal power flow problem (see e.g. [7], [8] or [1]), where now we deal with the allocation of both, power and AS at the same time. Tie-line constraints are here not taken into account, and the transmission system is considered to be robust enough and to have sufficient transmission capacity. In this study, we are concerned with one PTU of ahead markets only, and, therefore, time is not a variable in the considered problem formulation. Furthermore, each BRP is seen as a price taker, i.e., the influence of its bid is such that it cannot significantly change market outcomes and the global price of commodities. Through the rest of this paper we assume that there is one BRP in each node, and we use terms "node" and "BRP" interchangeably.

\footnotetext{
${ }^{5}$ TSO calculates this amount.
} 


\section{A. Coupling of Power and AS markets}

With the abbreviation $x:=\left[p^{\top} a^{\top}\right]^{\top}$, it is trivial to rewrite the objective function (1) of the OPRD problem in matrix form

$$
J(x)=x^{\top} H x+f^{\top} x
$$

with $H=\left[\begin{array}{cc}C_{n} & D_{n} \\ D_{n} & D_{n}\end{array}\right]$, where $C_{n}:=\operatorname{diag}\left(\alpha_{1}, \ldots, \alpha_{n}\right)$, $D_{n} \quad:=\operatorname{diag}\left(\mu_{1} \alpha_{1}, \ldots, \mu_{n} \alpha_{n}\right) ; \quad$ and $\quad f^{\top}=$ $\left[\beta_{1}, \ldots, \beta_{n}, \mu_{1} \beta_{1}, \ldots, \mu_{n} \beta_{n}\right]$. The above representation is a standard quadratic programming (QP) representation. The OPRD problem is suitable for QP solvers [9] under the assumption that all parameters, including confidential data that define cost and benefit functions, are known to some central entity. In a liberalized, market-based power systems, this is not the case. In this paper we treat the solution to the centralized QP-based OPRD problem as a reference for comparison with other proposed solutions.

The iterative bidding algorithms explained in Section IV-B and Section IV-C are devised with the aim that their solutions coincide with the solution of the work presented in Section III-B. When confidentiality, computing power and communication do not pose any constraints in finding a solution; the results obtained using a QP solver are the best solution achievable, and a "golden standard" for all the other solutions of the OPRD problem. In the remainder of the paper, we refer to it as the "golden standard" (GS) of the OPRD problem for the aforementioned reasons.

In practice different solution approaches have to be considered to cope with any assumption that cannot be fulfilled. For example, market organization can avoid dealing at some central level with confidentiality, distributed solution can cope with computing power and/or communication limitations, and also in certain cases with confidentiality issues.

In current market designs, BRPs have in principle one chance only to submit the bids for power and AS. To create these bids, due to coupling of power and AS markets, it is necessary to make assumptions on market outcome, i.e., realized prices on both markets. It is reasonable to assume that each BRP would be able to make bids for both, power and AS markets, that are economically more efficient ("better") if it was known in advance what the realized price on at least one of these markets would be.

The outcome of one market influences the outcome of the other market. Every bid curve is a result of a market outcome prediction, and, therefore; it depends on the forecast error. In turn, this error induces changes in the market clearing prices. Consequently, even in the conditions of perfect competition, the clearing prices cannot guarantee that the maximum of the social welfare is reached.

The challenge is to find new strategies that solve the above indicated problems. While doing so, it should also be kept in mind that confidentiality requirements, and not only necessary estimations from local level, pose serious burdens to the system. One possibility to overcome the above described difficulties is to create iterative markets. Iterative market arrangements not only remove the requirement that one single entity knows all the confidential data to reach the optimum, but also facilitate making approximations and enable BRPs making much better bids in each step of iteration.

We present two approaches for designing iterative markets. One design strategy is completely based on the dual decomposition and subgradient methods. It considers iterative bidding of amounts of power, $p_{i}$, and AS, $a_{i}$, for globally given prices $\lambda_{\mathrm{P}}$ and $\lambda_{\mathrm{A}}$. We refer to this approach as the "point bid-based iterative market" (PBM), and we present it in some more detail in Section IV-B. Alternative design strategy considers BRPs bidding of complete $\lambda_{\mathrm{P} i}\left(p_{i}\right)$ and $\lambda_{\mathrm{A} i}\left(a_{i}\right)$ curves, similarly as is done in the current practice, with the difference that that the bidding is performed in several iterations for each PTU. We refer to this approach as the "curve bid-based iterative market" $(\mathrm{CBM})$, and we present it in detail in Section IV-C.

\section{B. Golden Standard for Solutions of the OPRD Problem}

The quadratic programming problem is a special, widelyknown class of optimization problems; and the theory, including solution methods, and algorithms for QP problems are highly developed. It is a subclass of a more general class, convex optimization problems ${ }^{6}$. Once the problem is formulated in such a manner that it fits in the framework of convex optimization, there is a number of available convex optimization solvers that can be applied to solve it. The QP solver finds the optimal solution when the full knowledge of all the parameters of the objective function and constraints.

\section{ITERATIVE MARKET ARRANGEMENTS}

\section{A. Dual decomposition}

We present the market-based solution of the OPRD problem based on the Lagrangian relaxation. The constraints of the OPRD problem can be grouped into local and global constraints. Local constraints exist on a BRP level and all information needed for their satisfaction can be collected locally. An example of this type of constraints are lower and upper power production limits given by (2c). Equation (2d) also defines a local constraint. Global constraints, also called coupling or complicating constraints, are constraints that connect all producers and consumers in one system. For example, the power balance requirement, (2a), or requirement for certain amount of reserves in the system, (2b), are global constraints. Since, in a competitive market environment, it is highly unlikely that BRPs will share their local information with any other entity, the OPRD problem should be decomposed and solved in such a way that each BRP autonomously optimizes its own costs, while the overall system still reaches the global optimum. To accomplish this goal, we dualize only the coupling constraints by employing dual decomposition [10] and subgradient [11] methods. Decomposition not only removes the requirement for sharing the sensitive data but also significantly decreases the computational complexity. The partial Lagrangian consists of

\footnotetext{
${ }^{6}$ Note that this is the case only if $H$ defined in Section III-A is $H \succ 0$
} 
the global objective function (1) and global constraints (2a) and $(2 b)$, and is given by:

$$
\begin{aligned}
\mathcal{L}\left(p, a, \lambda_{P}, \lambda_{A}\right):= & \sum_{i=1}^{n} J_{i}\left(p_{i}, a_{i}\right)-\lambda_{P}\left(\sum_{i=1}^{n} p_{i}\right) \\
& -\lambda_{A}\left(\sum_{i=1}^{n} a_{i}-A_{\text {req }}\right) .
\end{aligned}
$$

The Lagrange multipliers $\lambda_{P}$ and $\lambda_{A}$ have an interpretation of global prices for energy and AS, respectively. Lagrange dual problem is given by:

$$
\max _{\lambda_{P}, \lambda_{A}} l\left(\lambda_{P}, \lambda_{A}\right)
$$

subject to

$$
\lambda_{A} \geq 0
$$

where $l\left(\lambda_{P}, \lambda_{A}\right)$ is the dual objective function defined as follows

$$
l\left(\lambda_{P}, \lambda_{A}\right):=\min _{p, a}\left\{\mathcal{L}\left(p, a, \lambda_{P}, \lambda_{A}\right)\right\}
$$

subject to $(2 \mathrm{c})$ and $(2 \mathrm{~d})$.

For fixed $\lambda_{P}, \lambda_{A}$, it is straightforward to decompose the dual function (4) and, as a result obtain $n$ decoupled local optimization problems, where each local optimization problem, which is defined as follows, is assigned to one node only.

\section{Problem IV.1 Local Optimization Problem}

Given the prices $\lambda_{P}$ and $\lambda_{A}$, for each $i \in\{1, \ldots, n\}$, the local optimization problem is given by

$$
\min _{p_{i}, a_{i}} g_{i}\left(\lambda_{P}, \lambda_{A}\right)
$$

subject to:

$$
\begin{aligned}
\underline{p_{i}}+a_{i} & \leq p_{i} \leq \overline{p_{i}}-a_{i}, \\
a_{i} & \geq 0
\end{aligned}
$$

where objective function is defined as $g_{i}\left(\lambda_{P}, \lambda_{A}\right):=$ $J_{i}\left(p_{i}, a_{i}\right)-\lambda_{P} p_{i}-\lambda_{A} a_{i}$.

For given $\lambda_{P}$ and $\lambda_{A}$, the minimizers $p_{i}^{*}$ and $a_{i}^{*}$ of the Local Optimization Problem (Problem IV.1) are the optimal values of power and AS in a node $i$.

The maximizers of the dual problem (4), $\lambda_{P}^{*}$ and $\lambda_{A}^{*}$, are the optimal market prices for power and AS. For the optimal market prices, power balance constraint and, (2a), and constraint on amount of AS, (2b), are necessarily satisfied [10], [12].

\section{B. Point Bid-Based Iterative Market (PBM)}

1) Formal representation: If we assume transparent markets, with prices $\lambda_{P}$ and $\lambda_{A}$ that are publicly (globally) available, the Problem IV.1 is easily solved by a QP solver.

Since the dual function is, in general, not a smooth function in $\lambda_{P}$ and $\lambda_{A}$, gradient methods are not applicable and, therefore; subgradient methods are to be used.

$$
\begin{aligned}
& \lambda_{P}^{(k+1)}=\lambda_{P}^{(k)}-\rho_{P}^{(k)}\left(\sum_{i=1}^{n} p_{i}\right) \\
& \lambda_{A}^{(k+1)}=\max \left(0, \lambda_{A}^{(k)}-\rho_{A}^{(k)}\left(\sum_{i=1}^{n} a_{i}-A_{\text {req }}\right)\right)
\end{aligned}
$$

Step sizes, $\rho_{P}^{(k)}$ and $\rho_{A}^{(k)}$, are chosen in such a way that convergence is obtained, see e.g. [11] or [12] for more detail and proofs. They can be constant or can change in each iteration. The convergence of subgradient methods is proven in [11] for several choices of step size, e.g. for constant step size, $\rho_{k}=\rho>0$. Some other step size examples for which convergence is guaranteed are series $\rho_{k}=\frac{q}{r+k}, \quad q>0, r \geq 0$, and $\rho_{k}=\frac{q}{\sqrt{k}}, \quad q>0$. The difference in prices between two iterations $k$ and $k+1$ for both commodities, power and AS, is used as a stopping criterion. When these values fall below certain predefined threshold $\epsilon$, the auction is stopped. Theoretically, the dual variables, i.e. the prices, are not monotonously converging. Still, this stopping criterion has been chosen as it is of the practical value for the implementation.

\section{Algorithm (PBM) \\ repeat until the stopping criterion is satisfied:}

i) Solve $n$ local problems (Problem IV.1) for fixed global parameters $\lambda_{P}^{(k)}$ and $\lambda_{A}^{(k)}$ calculated in the iteration $k$, or for given initial global parameters, i.e., prices $\lambda_{P}^{(0)}$ and $\lambda_{A}^{(0)}$

ii) Update prices according to (8a) and (8b)

2) Implementation: The iterative point bidding method starts with a market operator ${ }^{7}$ announcing the initial prices for power and ancillary services (initial conditions): $\lambda_{P}^{(0)}$ and $\lambda_{A}^{(0)}$. Given these prices, each BRP $(i=1 \ldots n)$ maximizes its profit. This corresponds to solving optimization subproblems, i.e., local objective functions, as defined in Problem IV.1, for given fixed prices for power and ancillary services. Results of these optimization algorithms, that is, optimal power and AS amounts of each BRP, are then bade again and a market operator announces a new set of prices. The process is repeated until the stopping criterion is satisfied.

Although, the algorithm is proven to converge, it might happen that the convergence is very slow, i.e., that the optimum is reached after large number of iterations. Moreover, even though the global constraints are satisfied in the optimal point, there is no guarantee that they are also satisfied in every iteration. This means that it is not possible to stop the process of optimization, i.e., the bidding iterations, before the optimum is reached. In case of doing so, it might happen that the markets will not be cleared, and there will be a scheduled

\footnotetext{
${ }^{7}$ By market operator, we mean PX for power markets and TSO for AS markets. Our problem formulation enables them to be one entity or separated entities, as long as they are synchronized in time and publish clearing results simultaneously.
} 
power imbalance, insufficient amount of AS, or both. This may impose a serious practical implementation issue for this market clearing strategy and is also the reason why we propose another iterative market clearing approach.

\section{Curve Bid-Based Iterative Market (CBM)}

1) Formal representation: The OPRD problem can be solved in another way, with BRPs bidding not only optimal amounts of power or AS, but the whole price - power profiles, $\lambda_{\mathrm{P} i}\left(p_{i}\right)$ and $\lambda_{\mathrm{A} i}\left(a_{i}\right)$. The core idea still comes from the decomposition methods, but the algorithm itself is slightly modified. Same as before, the OPRD problem is decomposed in $n$ smaller, simpler problems given by Local Optimization Problem IV.1. Optimal values $p_{i}^{*}$ and $a_{i}^{*}$ are calculated as a result of each of these optimizations. Unlike in PBM, BRPs now do not bid only these values, but each of them calculates its own optimal bid curve for both markets as follows:

$$
\begin{aligned}
\lambda_{i}\left(p_{i}\right) & =\frac{\partial J_{i}\left(p_{i}, a_{i}^{*}\right)}{\partial p_{i}} \\
\lambda_{i}\left(a_{i}\right) & =\frac{\partial J_{i}\left(p_{i}^{*}, a_{i}\right)}{\partial a_{i}} .
\end{aligned}
$$

While calculating the new bid, each BRP takes care of its own production limits, as expressed in $(2 \mathrm{c})$. Note that with quadratic cost/benefit functions, the slope of each BRP's bid in every iteration is the same, but the production (consumption) low and high limits differ from one iteration to the other. After optimal bids are calculated, bid curves for power and AS markets are aggregated, the markets are cleared, and the new clearing prices are announced. The process repeats till the stopping criterion is satisfied.

In this paper, we present simulation results which indicate that the proposed algorithm converges to the optimal point. In some cases, however, the proposed algorithms runs into infeasible problems. This might be due to small number of BRPs that participate in the simulated market. Recursive feasibility and further analytic treatment of the algorithm are topics for future research.

\section{Algorithm (CBM) \\ repeat until the stopping criterion is satisfied: \\ i) Calculate the optimal bids for this step (for fixed global parameters $\lambda_{P}^{(k)}$ and $\lambda_{A}^{(k)}$ calculated in the previous iteration $k$, or for given initial global parameters, i.e., prices $\lambda_{P}^{(0)}$ and $\lambda_{A}^{(0)}$ ) according to (IV-C1) \\ ii) Sum up all bids for power and for AS and clear the markets (i.e., calculate the prices for which constraints (2a),(2b) are satisfied); update the prices}

2) Implementation: It is straightforward to verify that in each iteration all system constraints, local and global, are satisfied. Because the procedure, which is in essence equal to market clearing procedure, is done in each iteration step, it is guaranteed that all the constraints are met. This fact has the following advantageous practical implication: it is now possible to break the execution of the iterative optimization before actually reaching the optimum without violating the system's constraints.

In simulations it has been observed that in some cases, depending on choice of initial prices or market outcome in one of iterations, it could happen that ratio of prices is so unfavorable for AS that there are not enough bids to clear the AS market. This is due to taking local constraints into account only on local and not as well global level. In that sense, this algorithm is not robust.

\section{Simulation RESUlTS AND DISCUSSION}

Simulations are run in Matlab on a model of 12 nodes devised from Table 1. of [13] where typical range of power market parameters is listed. Simulations are run for several cases, but only one of them is presented here as similar conclusions hold for all of them. The simulation parameters are given in Table I. In the presented case, the required amount

TABLE I

Simulation PARAMETERS

\begin{tabular}{ccccc}
\hline \hline BRP & $\alpha$ & $\beta$ & $\underline{p_{i}}$ & $\overline{p_{i}}$ \\
\hline 1 & 0.008 & 40.0 & 0 & 155 \\
2 & 0.009 & 46.0 & 0 & 190 \\
3 & 0.012 & 44.0 & 0 & 150 \\
4 & 0.012 & 45.0 & 0 & 150 \\
5 & 0.011 & 43.0 & 0 & 140 \\
6 & -0.008 & 50.0 & -150 & 0 \\
7 & -0.010 & 48.0 & -150 & 0 \\
8 & -0.011 & 47.0 & -150 & 0 \\
9 & -0.014 & 49.0 & -150 & 0 \\
10 & -0.012 & 47.5 & -150 & 0 \\
11 & -0.008 & 50.0 & -150 & 0 \\
12 & -0.015 & 47.0 & -150 & 0 \\
\hline \hline
\end{tabular}

of reserves is chosen to be $A_{\text {req }}=120$ and $\mu=0.06$ for all BRPs. To facilitate the comparison of algorithms, the same initial condition and stopping criterion are implemented in both algorithms.

Optimal prices are calculated to be $\lambda_{P}^{*}=46.68$ and $\lambda_{A}^{*}=2.82$. The results of the simulations of both market arrangements (PBM and CBM), are given in Figure 1 and Figure 2. First observation is that, indeed, both methods converge to the real optimum, i.e., the solution obtained by GS. PBM procedure reached the optimum after $k=116$ iterations, while it took only $k=16$ iterations for CBM to find it.

The PBM method has proven to be very sensitive to the choice of the step size update $\rho_{P}^{(k)}$ and $\rho_{A}^{(k)}$, see equation (8a) and (8b). In case of choosing too small step size, the convergence might be very slow, or, in case of too big parameters $\rho_{P}^{(k)}$ and $\rho_{A}^{(k)}$, Lagrange multipliers, i.e. prices, could be too oscillatory. The speed of convergence also depends on the choice of initial prices.

However, while PBM has shown to be significantly dependant on choice of $\rho_{P}^{(k)}$ and $\rho_{A}^{(k)}$ which can lead to slowness and oscillatory behavior, it has also proven to be more robust with respect to choosing the initial prices compared to CBM. 

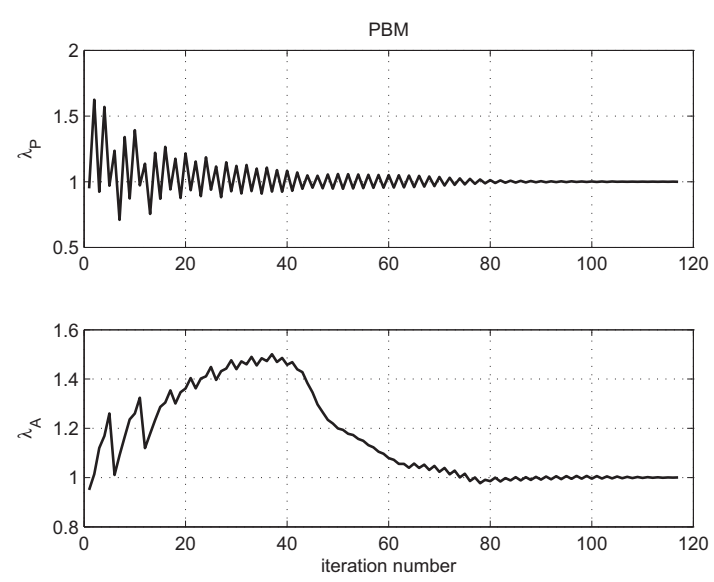

Fig. 1. Normalized prices for power and AS on PBM markets.
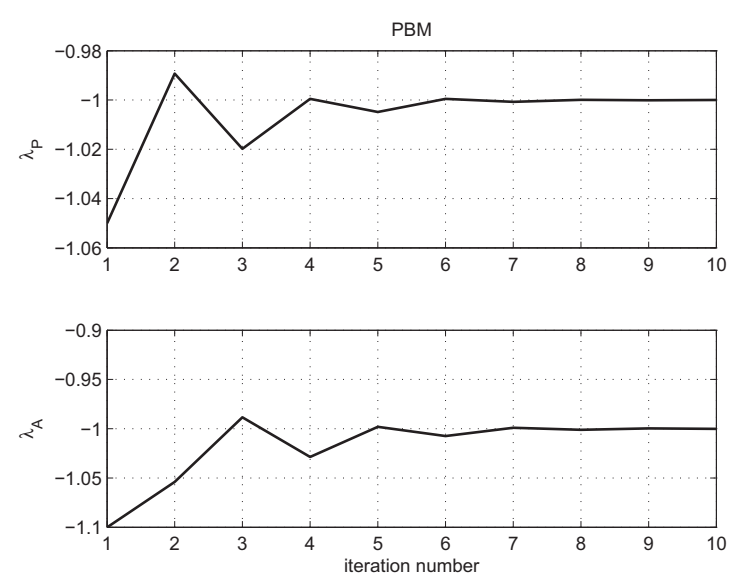

Fig. 2. Normalized prices for power and AS on CBM markets.

\section{CONCLUSIONS}

In this paper, we studied the interaction of power and AS markets and investigated different approaches of integrating these markets. It has been shown that in current market auctions the optimal social welfare is, in general, not reached even under conditions of the perfect market competition, and that BRPs are not available to offer bids to the market in such way that the maximal economic efficiency is achieved.

In this paper, we suggested and analyzed two new iterative power and AS market designs. They are both based on decomposition methods, with the important difference that in one case (PBM) BRPs bid only optimal values of power and AS, while in the other case (CBM) the the BRPs submit complete curves that describe dependencies between power and price. In the first case, the global constraints are fulfilled only in the optimal point. In the second case, all the constraints are satisfied in every iteration and if the optimization is stopped before actually reaching the optimum, the only property lost is optimality itself.

Simulation results show some weak points of the PBM design, such as possible slow convergence and great oscil- lations in prices. Still, for this market design, convergence is analytically proven, unlike that for the CBM design. The CBM markets converge in the significantly smaller number of iterations, but on the downside, the method is sensitive to a choice of the initial market prices. However, in both designs the effects of errors in prediction of market outcome for BRPs are removed and the overall social welfare is increased when compared to today's situation.

\section{ACKNOWLEDGMENT}

This work was supported by the European Commission Research Project FP7-ICT-249096 Price-based Control of Electrical Power Systems (E-Price), www.e-price-project.eu.

\section{REFERENCES}

[1] S. Stoft, Power Economics. San Francisco: Ignatius Press, 2002.

[2] A. Jokić, "Price-based optimal control of electrical power systems," Ph.D. dissertation, Eindhoven, University of Technology, The Netherlands, 2007.

[3] X. Ma, D. Sun, and K. Cheung, "Energy and reserve dispatch in a multizone electricity market," Power Systems, IEEE Transactions on, vol. 14, no. 3, pp. 913-919, 1999.

[4] K. Cheung, X. Ma, and D. Sun, "Functional Design of Ancillary Service Markets Under the Framework of Standard Market Design for ISO New England," 2006 International Conference on Power System Technology, 2006.

[5] F. Stacke and P. Cuervo, "Integrated Pool/Bilateral/Reserve market operation under pay-as-bid pricing," 2008 5th International Conference on the European Electricity Market, pp. 1-8, 2008.

[6] A. Motto, F. Galiana, A. Conejo, and M. Huneault, "On walrasian equilibrium for pool-based electricity markets," IEEE Transactions on Power Systems, 2002.

[7] P. Kundur, Power System Stability and Control. New York: McGrawHill, 1994.

[8] F. C. Schweppe, M. C. Caramanis, and R. D. Tabors, Spot Pricing of Electricity (Power Electronics and Power Systems). Springer, 1988.

[9] S. Boyd and L. Vandenberghe, Convex Optimization. Cambridge University Press, 2004.

[10] S. Boyd, L. Xiao, A. Mutapcic, and J. Mattingley, "Notes on decomposition methods," 2007. [Online]. Available: http://www.stanford.edu/class/ee364b/notes/decomposition_notes.pdf

[11] S. Boyd and A. Mutapcic, "Subgradient methods," $2008 . \quad$ [Online]. Available: http://www.stanford.edu/class/ee364b/lectures/subgrad_method_notes.pdf

[12] D. P. Bertsekas, Nonlinear Programming. Athena Scientific, 1999.

[13] F. Alvarado, J. Meng, C. DeMarco, and W. Mota, "Stability analysis of interconnected power systems coupled with market dynamics," IEEE Transactions on Power Systems, 2001. 